\title{
Variations in human pulmonary vein ostia morphology - a systematic review with meta-analysis
}

\author{
Michał Bonczar ${ }^{1}$, Katarzyna Piątek-Koziej ${ }^{1}$, Joanna Wolska ${ }^{1}$, Olaf Tomala ${ }^{1}$, El-Ayachi \\ Stitou $^{1}$, Jakub Pękala ${ }^{1}$, Przemysław Pękala ${ }^{1}$, Jerzy Walocha ${ }^{1}$, Mateusz Hołda ${ }^{2}$, and \\ Mateusz Koziej ${ }^{1}$ \\ ${ }^{1}$ Uniwersytet Jagiellonski w Krakowie Katedra Anatomii \\ ${ }^{2}$ HEART - Heart Embryology and Anatomy Research Team Department of Anatomy \\ Jagiellonian University Medical College Cracow Poland
}

February 14, 2022

\begin{abstract}
Introduction: The aim of this study was to establish the most accurate and up-to-date anatomical knowledge of PVs ostia variations, diameters and ostial area, in order to provide physicians, especially heart and thoracic surgeons with exact knowledge concerning this area. Materials and methods: Major online medical databases such as PubMed, Embase, Scopus, Web of Science and Google Scholar were searched to gather all studies in which the variations, maximal diameter and ostial area of the PVs were investigated. During the study, the Preferred Reporting Items for Systematic Reviews and Meta-Analyses (PRISMA) guidelines were followed. Additionally, The Critical Appraisal Tool for Anatomical Meta-analysis (CATAM) was used to provide the highest quality findings. Reults: The most common ostia variation is classical one, containing left superior pulmonary vein (LSPV), left inferior pulmonary vein (LIPV), right superior pulmonary vein (RSPV) and right inferior pulmonary vein (RIPV). Mean diameters and ostial areas of each pulmonary vein were established in general population and in multiple variations considering method of gathering the data and geographical location. Conclusion: Significant variability in PV ostia is observed. Left-sided PVs have smaller ostia than corresponding right-sided PVs, and the inferior PVs ostia are smaller than superior. The size of the LCPV ostium is the largest among all analyzed veins, while the ostium of RMPV is the smallest. It is hoped that the results from this meta-analysis will help clinicians in planning and performing procedures that involve pulmonary and cardiac area.
\end{abstract}

Title: Variations in human pulmonary vein ostia morphology - a systematic review with metaanalysis

Authors: Michał Bonczar ${ }^{1} \mid$ Katarzyna Piątek-Koziej $^{1} \mid$ Joanna Wolska $^{1} \mid$ Olaf Tomala $^{1} \mid$ El-Ayachi Stitou ${ }^{1}$ | Jakub Pękala ${ }^{1}$ Przemysław Pękala ${ }^{1}$ | Jerzy Walocha ${ }^{1} \mid$ Mateusz Hołda ${ }^{2,3}$ | Mateusz Koziej ${ }^{1,2}$

Affiliations:

${ }^{1}$ Department of Anatomy, Jagiellonian University Medical College, Kraków, Poland

${ }^{2}$ HEART - Heart Embryology and Anatomy Research Team, Department of Anatomy, Jagiellonian University Medical College, Cracow, Poland

${ }^{3}$ Division of Cardiovascular Sciences, The University of Manchester, UK

Corresponding author: Michał Bonczar, Department of Anatomy, Jagiellonian University Medical College, Mikołaja Kopernika 12, 33-332 Kraków, Poland. Email: michalbonczar01@gmail.com. 
Conflicting interests: The author(s) declare no potential conflicts of interest with respect to the research, authorship, and/or publication of this article.

Funding: The author(s) received no financial support for the research, authorship, and/or publication of this article.

Informed consent: Not applicable

Ethical approval: Not applicable

Title: Variations in human pulmonary vein ostia morphology - a systematic review with metaanalysis

\begin{abstract}
:
Introduction: The aim of this study was to establish the most accurate and up-to-date anatomical knowledge of PVs ostia variations, diameters and ostial area, in order to provide physicians, especially heart and thoracic surgeons with exact knowledge concerning this area.Materials and methods: Major online medical databases such as PubMed, Embase, Scopus, Web of Science and Google Scholar were searched to gather all studies in which the variations, maximal diameter and ostial area of the PVs were investigated. During the study, the Preferred Reporting Items for Systematic Reviews and Meta-Analyses (PRISMA) guidelines were followed. Additionally, The Critical Appraisal Tool for Anatomical Meta-analysis (CATAM) was used to provide the highest quality findings.Reults: The most common ostia variation is classical one, containing left superior pulmonary vein (LSPV), left inferior pulmonary vein (LIPV), right superior pulmonary vein (RSPV) and right inferior pulmonary vein (RIPV). Mean diameters and ostial areas of each pulmonary vein were established in general population and in multiple variations considering method of gathering the data and geographical location.Conclusion: Significant variability in PV ostia is observed. Left-sided PVs have smaller ostia than corresponding right-sided PVs, and the inferior PVs ostia are smaller than superior. The size of the LCPV ostium is the largest among all analyzed veins, while the ostium of RMPV is the smallest. It is hoped that the results from this meta-analysis will help clinicians in planning and performing procedures that involve pulmonary and cardiac area.
\end{abstract}

Key Words: pulmonary veins variations, pulmonary veins ostia, pulmonary veins diameter, atrial fibrillation, catheter ablation.

\title{
1 | INTRODUCTION
}

Pulmonary veins (PVs) are blood vessels which distribute oxygenated blood from lungs to the left atrium (LA) of the heart. Blood inflow to the LA is generally described as a system of four PVs, arranged in pairs, each located on the right and left side of the LA. The four most commonly occurring vessels are left superior pulmonary vein (LSPV), left inferior pulmonary vein (LIPV), right superior pulmonary vein (RSPV) and right inferior pulmonary vein (RIPV) (classical type). However, a substantial number of variations in quantity, arrangement and morphometric dimensions of the PVs can be observed. Atrial fibrillation (AF) is the most common arrhythmia, and its incidence increases significantly with patients' age . Both PVs and the LA play a significant role in the pathogenesis of AF. Myocardial sleeves of the distal PVs form simple extensions of the left atrial myocardium over the outer surface of PVs and they are the major source of ectopic beats. A detailed anatomical knowledge about PVs can be useful while preforming catheter ablation in the treatment of AF, may result in higher effectiveness of surgical therapies and can aid radiological examination of the pulmonary and heart area. Therefore, the aim of this study was to establish the most accurate and up-to-date anatomical knowledge of PVs ostia variations, diameters and ostial area.

\section{2 | MATERIALS AND METHODS}

\subsection{Search strategy}

Study search and data collection was performed from June to September 2021. Major online medical databases such as PubMed, Embase, Scopus, Web of Science and Google Scholar were searched to gather all 
studies in which the variations, maximal diameter and ostial area of the PVs were investigated. The following search terms were used utilizing the Boolean technique: (pulmonary veins anatomy OR pulmonary veins anatomy variation OR accessory pulmonary veins OR anomalous pulmonary venous return OR pulmonary veins origin) AND (pulmonary veins ostial area) AND (pulmonary veins diameter OR pulmonary veins width). Neither date, language, article type nor text availability conditions were applied. An additional search through the references of the identified studies was conducted at the end of the search stage to ensure the accuracy of the process. During the study, the Preferred Reporting Items for Systematic Reviews and Meta-Analyses (PRISMA) guidelines were followed. Additionally, The Critical Appraisal Tool for Anatomical Meta-analysis (CATAM) was used to provide the highest quality findings .

\section{2 | Eligibility assessment}

The database search and the manual search identified a total of 9119 studies which were initially evaluated by two independent reviewers. After removing the duplicates and irrelevant records, a total of 1573 articles were qualified for a full-text evaluation. To minimize the potential bias and to maintain an accurate statistical methodology, articles such as case reports, case series, conference reports, reviews, letters to the editors and studies that provided incomplete or irrelevant data were excluded. Inclusion criteria involved original studies with extractable numerical data regarding variations, maximal diameter and ostial area of the PVs. As a result, 73 studies were included for variations analysis, 86 for diameter analysis and 18 for ostial area analysis. The AQUA Tool which was specifically designed for anatomical meta-analyses was used to minimize the potential bias of included studies. The flowchart outlining the study inclusion process is shown in Figure 1.

\section{3 | Data extraction}

Data from studies that met inclusion criteria were extracted by two independent reviewers. Qualitative data, such as year of publication, country and continent of origin, methodology of data collection, information about the diseases in the studied groups were gathered. Quantitative data, such as sample size, numerical data regarding prevalence among variations of the PVs, mean maximal diameter of every PV with its standard deviation or interquartile range and mean ostial area of every PV with its standard deviation or interquartile range were also extracted. Studies containing mean results, but without standard deviation or interquartile range or unclear or unspecified variations were excluded. Any discrepancies between studies identified by the two reviewers were resolved by contacting the authors of the original studies wherever possible or by consensus with a third reviewer.

\subsection{Statistical analysis}

To perform the meta-analyses, STATISTICA version 13.1 software (StatSoft Inc., Tulsa, OK, USA), MetaXL version 5.3 software (EpiGear International Pty Ltd, Wilston, Queensland, Australia) and Comprehensive Meta-analysis version 3.0 software (Biostat Inc., Englewood, NJ, USA) were used. A random-effects model was used in all analyses. Chi-squared test and I-squared statistic were employed to assess the heterogeneity among the studies. A p-value and confidence intervals were used to determine statistical significance between studies. A p-value lower than 0.05 was considered statistically significant. In case of overlapping confidence intervals, differences were considered statistically insignificant. I-squared statistics were interpreted as follows: values of $0-40 \%$ were considered as "might not be important", values of $30-60 \%$ were considered as "might indicate moderate heterogeneity", values of 50-90\% were considered as "may indicate substantial heterogeneity" and values of 75-100\% were considered as "may indicate substantial heterogeneity".

\section{3 | RESULTS}

\section{1 | Anatomical variations}

Studies that matched the required criteria and contained both complete and relevant data were included in the anatomical variations part of this meta-analysis. Basic characteristics of all 73 included studies are presented in Table 1. Anatomical variations were collected using a two-stage compilation method, established by authors of this study, based on the most common variations described in the literature. In stage one, data 
from qualified studies were divided into two groups: subjects who (1) had classical arrangement of the PV confluence or (2) had any other arrangement than classical type. As classical type (Type 1) authors consider two main PVs on the right side and two on the left side: RSPV, RIPV, LSPV and LIPV. In the second stage, authors established 9 subtypes of arrangements of the PVs, that differed from the classical one: Type 2 arrangement contains RSPV, RIPV and one left common pulmonary vein (LCPV); Type 3 - arrangement contains LSPV, LIPV and on right common pulmonary vein (RCPV); Type 4 - arrangement contains RSPV, RIPV, LSPV, LIPV and an additional right middle pulmonary vein (RMPV); Type 5 - arrangement contains RSPV, RIPV, LSPV, LIPV and both additional RMPV and LMPV; Type 6 - arrangement contains LSPV, LIPV, LMPV and one RCPV; Type 7 - arrangement contains RSPV, RIPV, LSPV, LIPV and an additional left middle pulmonary vein (LMPV); Type 8 - arrangement contains RSPV, RIPV, RMPV and one LCP; Type 9 - arrangement contains one LCPV and one RCPV; Type 10 - includes all other arrangements. The analysis of the 9 types, different than the classical one, was performed using only those studies that clearly differentiated those arrangements. Imaged arrangements according to authors compilation method are shown in Figure 2.

Overall, the anatomical variations of PV ostia were examined in 73 studies including 13811 subjects . The pooled prevalence of classical type was $70.25 \%$ (95\% CI: $66.05 \%-74.28 \%)$. The pooled prevalence of any other type was $29.50 \%$ (95\% CI: $25.20 \%$ - 33.99\%). It may be concluded that data regarding those two types may represent considerable heterogeneity as the $\mathrm{I}^{2}$ was established appropriately 96.28 for the classical type and 96.77 for other types. The pooled prevalence of 9 other types were established based on 8504 subjects and were found to be $17.37 \%$ (95\% CI: $14.67 \%$ - 20.25\%) for type 2, $8.78 \%$ (95\% CI: $6.03 \%$ - $11.98 \%$ ) for type 3, $8.38 \%$ (95\% CI: $6.27 \%-10.76 \%$ ) for type $4,5.11 \%$ (95\% CI: $3.87 \%-6.51 \%)$ for type $5,1.24 \%(95 \%$ CI: $0.72 \%-1.89 \%)$ for type $6,1.19 \%$ (95\% CI: $0.73 \%-1.75 \%)$ for type $7,1.09 \%$ (95\% CI: $0.64 \%-1.66 \%)$ for type $8,0.20 \%$ (95\% CI: $0.12 \%-0.31 \%$ ) for type 9 and $5.29 \%$ (95\% CI: $4.08 \%$ - 6.65\%) for type 10 (Table 2). The data regarding types $2,3,4,5,6,8$ and 10 may represent considerable heterogeneity as the established $\mathrm{I}^{2}$ were included in the compartment from 75 to 100 (Table 2).

The studies that were carried out on patients with AF were subjected to additional analysis. The pooled prevalence in patients with AF was calculated based on 11103 subjects and was found to be $70.61 \%$ (95\% CI: $65.55 \%-75.42 \%$ ) for classical arrangement of PVs and 29.05\% (95\% CI: $23.92 \%-34.45 \%$ ) for any other type (Table 2). It may be concluded that data regarding those types may represent considerable heterogeneity as the established $\mathrm{I}^{2}$ were 96.81 for classical type and 97.23 for any other than classical type. The most common variation regarding all other arrangements than the classical one in patients with AF was found to be Type 2 (RSPV, RIPV and LCPV) and its pooled prevalence was $18.16 \%$ (95\% CI: $15.30-21.21 \%$ ) (Table 2). When comparing PV ostia variations between general population and AF patients, no statistically significant differences were found in the distribution of the classical type $(\mathrm{p}=0.92)$. More thorough analyses show no significant differences in subtypes distribution with the types with either additional middle PV ostium on left or right or both sides (Types 4-8) patients with and without AF ( $p>0.05$ ). For details, please see Tables 2 and 3 .

Included studies differed in a way of imaging the number and the arrangement of the pulmonary veins. Computed tomography, echocardiography, magnetic resonance imaging, magnetic resonance angiography, intraoperative imaging and cadaver dissection methods were used in analyzed studies to establish PVs arrangements. Hence, an additional statistical analysis according to imaging technique was performed (no significant differences, all $\mathrm{p}>0.05$ ). Also, a further analysis according to geographical location of each study was performed. For details, please see Table 2.

3.2 | PVs ostia diameters

Mean maximal diameters of PVs ostia were extracted from 86 studies that matched the inclusion criteria . Baseline characteristics of each included study are gathered in Table 4. Data regarding mean maximal diameters of 6 most commonly occurring PV ostia: RSPV, RIPV, LSPV, LIPV, RMPV and LCPV are shown in Table 5. All data concerning diameters were either presented or have been converted by authors of this study to millimeters [mm]. Mean maximal diameter of RSPV was established based on 12073 subjects 
and was found to be $18.87 \mathrm{~mm}$ (95\% CI: $18.35-19.40 \mathrm{~mm}$ ) and mean maximal diameter of RIPV ( $\mathrm{n}=11980)$ was $17.17 \mathrm{~mm}$ (95\% CI: $16.65-17.69 \mathrm{~mm}$ ). Mean maximal diameter of LSPV was established based on 11865 subjects and was found to be $18.12 \mathrm{~mm}$ ( $95 \%$ CI: $17.63-18.61 \mathrm{~mm})$, while the mean maximal diameter of LIPV ( $\mathrm{n}=11849$ ) was $16.25 \mathrm{~mm}$ (95\% CI: $15.82-16.69 \mathrm{~mm}$ ). The comparison between individual PV ostia shows that RSPV ostium is significantly larger than RIPV (Table 5). The same was observed for leftsided PV ostia (superior larger than inferior) (Table 5). Left-sided PV ostia were observed to have smaller diameters than corresponding right-sided PVs; however, the difference was not statistically significant (Table 5). Mean maximal diameter of RMPV was established based on 5030 subjects and was found to be $9.43 \mathrm{~mm}$ (95\% CI: $8.89-9.96 \mathrm{~mm}$ ) and was the smallest among analyzed ostia. Mean maximal diameter of LCPV was established based on 4966 subjects and was found to be $29.01 \mathrm{~mm}$ (95\% CI: $28.07-29.94 \mathrm{~mm}$ ) (Table $5)$, which was the largest value among all analyzed veins.

Included studies significantly differed in methodology of measuring the maximal diameter of each PV ostium (angiography, computed tomography, echocardiography, magnetic resonance imaging and magnetic resonance angiography methods). Hence, additional statistical analyses of data according to imaging technique and geographical location were performed (Table 5).

\section{3 | Ostial area}

The data on ostial area were extracted from 18 studies that matched the inclusion criteria . Baseline characteristics of each analyzed study are gathered in Table 6. Data regarding mean ostial area of 6 most common PV ostia: RSPV, RIPV, LSPV, LIPV, RMPV and LCPV are shown in Table 7. All data regarding ostial area were either presented or have been converted by authors of this study to square millimeters $\left[\mathrm{mm}^{2}\right]$. Mean ostial area of RSPV was established based on 2697 subjects and was found to be $280.12 \mathrm{~mm}^{2}$ (95\% CI: 253.25 - $\left.307.01 \mathrm{~mm}^{2}\right)$ and mean ostial area of RIPV $(\mathrm{n}=2697)$ was $232.75 \mathrm{~mm}^{2}\left(95 \%\right.$ CI: $\left.214.45-251.05 \mathrm{~mm}^{2}\right)$. Mean ostial area of LSPV was established based on 2624 subjects and was found to be $228.27 \mathrm{~mm}^{2}(95 \%$ CI: $210.53-246.01 \mathrm{~mm}^{2}$ ) and mean ostial area of LIPV $(\mathrm{n}=2464)$ was $175.99 \mathrm{~mm}^{2}$ (95\% CI: $162.94-189.05$ $\mathrm{mm}^{2}$ ). The comparison between individual PV ostia indicates that RSPV ostium area is significantly larger than RIPV (Table 7). The same was observed for left-sided PVs ostial area (superior greater than inferior) (Table 7). Moreover, the difference between left-sided and right-sided veins was observed (right ostial areas larger than left) (Table 7). Mean ostial area of RMPV was established based on 1285 subjects and was found to be $68.01 \mathrm{~mm}^{2}$ (95\% CI: $54.11-81.91 \mathrm{~mm}^{2}$ ), which was the smallest area among all analyzed vein ostia. Mean ostial area of LCPV was established based on 1269 subjects and was found to be $451.92 \mathrm{~mm}^{2}(95 \%$ CI: $388.50-515.35 \mathrm{~mm}^{2}$ ) (Table 7), which was the largest observed area (statistically significant).

Included studies differed in a way of imaging the ostial area of each pulmonary vein. Computed tomography, magnetic resonance imaging and magnetic resonance angiography methods were used to establish those areas. Hence, additional statistical analyses of data according to imaging technique and location were performed. All results mentioned above and more detailed statistics are presented in Table 7.

\section{4 | DISCUSSION}

Although a large number of studies has been published on variations of the PVs ostia, their results are often contradictory and precise prevalence of each variation has not been established yet. Therefore, the aim of this meta-analysis was to provide a detailed, more precise and up-to-date anatomical knowledge base. An accurate understanding of the anatomy is important for clinicians as it may greatly increase a chance of successful therapy. Gathering and analyzing data published in the literature before, allowed us to establish more precise and statistically significant prevalence for each variation type (Figure 2).

Moreover, homogeneity between the general group and the patients with AF was established. P-value for classical type was 0.92 and for other than classical types was 0.90 . Hence, it may be concluded that there are no statistically significant differences between the general group and patients with AF. Additionally, a comparison between patients with and without AF was made. All obtained p-values (Table 3) are higher than 0.05, except for types 5 (other arrangements) and 10 (LCPV and RCPV). Therefore, it may be assumed that, in a great majority, there are no statistically significant differences in PV anatomy between patients 
with and without AF. However, it must be noted that abundance and $\mathrm{I}^{2}$ of those two groups are strongly different. Hence, those results are burdened with a potential bias, which can be eliminated in further papers, where a larger group of patients with AF will be studied, and if the results will have been showing greater heterogeneity. Furthermore, maximal diameter and ostial area of PVs also indicate substantial variability. The mean maximal diameters range from $10.9 \mathrm{~mm}$ to $27.7 \mathrm{~mm}$ for RSPV, from $7.5 \mathrm{~mm}$ to $23 \mathrm{~mm}$ for RIPV, from $10.0 \mathrm{~mm}$ to $25.0 \mathrm{~mm}$ for LSPV, from $8.0 \mathrm{~mm}$ to $21.5 \mathrm{~mm}$ for LIPV, from $6.9 \mathrm{~mm}$ to $17.0 \mathrm{~mm}$ for RMPV and from $18.7 \mathrm{~mm}$ to $38.2 \mathrm{~mm}$ for LCPV. Regarding ostial area of each PV, the values range from $132.2 \mathrm{~mm}^{2}$ to $377.0 \mathrm{~mm}^{2}$ for RSPV, from $142.6 \mathrm{~mm}^{2}$ to $298.0 \mathrm{~mm}^{2}$ for RIPV, from $106.5 \mathrm{~mm}^{2}$ to $317.3 \mathrm{~mm}^{2}$ for LSPV, from $98.0 \mathrm{~mm}^{2}$ to $248.2 \mathrm{~mm}^{2}$ for LIPV, from $36.2 \mathrm{~mm}^{2}$ to $123.6 \mathrm{~mm}^{2}$ for RMPV and from 308.2 $\mathrm{mm}^{2}$ to $694.0 \mathrm{~mm}^{2}$ for LCPV.

$\mathrm{AF}$ is the most common cardiac rhythm disturbance and is responsible for substantial morbidity and mortality in general population. PVs play a crucial role in AF development . Catheter ablation is a standard treatment for patients with $\mathrm{AF}$, which primarily focuses on electrical isolation of the pulmonary veins . It is a very promising procedure, as it shows a great efficacy rate; however, a detailed knowledge and awareness of patients' PV arrangement and their morphological parameters are essential for safe and successful performance. Collecting and analyzing the data gathered in the literature allowed us to establish the most accurate values of the ostial area and its diameter. Narrower PVs pose a greater challenge when performing a catheter ablation. Therefore, the results from this meta-analysis may be a helpful tool for surgeons performing this procedure. Detailed and accurate anatomical knowledge combined with an appropriate pre-procedural imagining may strongly increase chances of successful therapy.

A detailed and precise anatomical knowledge is also essential when performing other cardiothoracic procedures. The PVs are often overlooked structures in chest imaging, and can become directly or indirectly involved in a wide array of pathological and nonpathological processes ranging from malignant involvement to surgical changes to differences in anatomic arrangement. Although PV variations are frequently asymptomatic, the knowledge of these variations is important while planning cardiothoracic surgeries, as it may prevent potential complications . Therefore, physicians, especially radiologists, should be aware of them, as appropriate pre-procedural imagining may increase the chances of successful therapy.

Choosing an appropriate method to establish patients' individual internal anatomy may also play a significant role in clinical practice. In included studies, most frequently used ways of imaging PV anatomical variations were computed tomography scans, magnetic resonance imaging, magnetic resonance angiography and echocardiography. However, there are no statistically significant differences in imaging method of the observed PV anatomical variations (all $\mathrm{p}>0.05$ ). The most frequently used method of establishing the maximal diameter of each PV were angiography, computed tomography, magnetic resonance imaging, magnetic resonance angiography and echocardiography. Comparison of the groups showed statistically significant difference between echocardiography and the rest of the methods (all $\mathrm{p}<0.05$ ), with echocardiography reporting the smallest values (Table 5). Therefore, it may concluded that the values of maximal PV diameter obtained using echocardiography differ from the results obtained by other methods (as they are underestimated). Consequently, echocardiography should not be recommended for establishing PV diameter. The most frequently used method of establishing the ostial area were computed tomography, magnetic resonance imaging and magnetic resonance angiography. Comparison of the groups showed no statistically significant differences between results obtained by those methods (all $\mathrm{p}>0.05$ ) (Table 7 ).

Human anatomy can potentially vary in different regions of the world (geographical and ethnical differences). The results obtained in different continents were compared in order to establish the potential heterogeneity among them. With regards to the PV ostia anatomical variations, maximal PV ostia diameter and PV ostial area, there were no statistically significant differences between continents, as all the p-values were significantly higher than 0.05 (Table 2,5 and 7 ).

This study has several limitations. First of all, all limitations in the current study are the reflection of many flaws of individual studies included in the meta-analyses. The majority of our results were determined by the rate of significant heterogeneity. Although many subgroup analyses were conducted, the potential sources 
of heterogeneity could not be indicated. The measurement site and the method of collecting the PV ostium maximal diameter and ostial area may also significantly differ between individual studies. Furthermore, the RCPV ostial diameter and ostial area were not established due to limited and inconsistent amount of data in the literature. Due to insufficient number of studies, we were not able to perform subgroups analyses targeting other disease entities than AF. Furthermore, the studies investigating variations, diameter and ostial area of the PVs were mostly carried out in Asia, Europe and North America. This can be a potential source of bias as our results reflect Asian, European and Northern American people rather than the global population. Further anatomical studies should be conducted to investigate these features in a larger and more ethnically diverse group of patients.

In conclusion, this is the most comprehensive and up-to-date study regarding PV ostia variability and their size. Several types of the PV ostia arrangement may be observed with the classical type being the most popular (70\%), followed by the type with common ostium for left-sided PVs (17\%), type with common ostium for right-sided PVs (9\%) and type with additional middle PV on the right side (8\%). Significant differences in PV ostia variations are observed in AF patients with more prevalent types with either additional middle PV ostium on the left or right or both sides. A significant variability in PV ostia is observed. Left-sided PVs have smaller ostia than corresponding right-sided PVs, and the inferior PVs ostia are smaller than superior. The size of the LCPV ostium is the largest among all analyzed veins, while the ostium of RMPV is the smallest. It is hoped that the results from this meta-analysis will help clinicians in planning and performing procedures that involve pulmonary and cardiac area.

\section{5 | REFERANCES}

\section{LEGENDS:}

Figure 1 Flow diagram presenting process of collecting data included in this meta-analysis.

Figure $2 \mid$ A - Classical Type. B - Type 2. C - Type 3. D - Type 4. E - Type 5. F - Type 6. G - Type 7. H Type 8. I - Type 9.

Table 1 | Characteristics of each study included in variations part of this meta-analysis.

Table 2 | Statistical results in variations part of this meta-analysis. AF - patients with atrial fibrillation. Non-AF - patients without atrial fibrillation. CT - computed tomography. Echo - echocardiography. MRI - magnetic resonance imaging. MRA - magnetic resonance angiography. Surgery - data established intraoperatively. Cadavers - data established during cadaver dissection. LCL - lower confidence interval. HCL higher confidence interval. Q - Cochrain's Q. RSPV - right superior pulmonary vein. RIPV - right inferior pulmonary vein. LSPV - left superior pulmonary vein. LIPV - left inferior pulmonary vein. RMPV - right middle pulmonary vein. LMPV - left middle pulmonary vein. LCPV - left common pulmonary vein. RCPV - right common pulmonary vein.

Table 3 | P-values obtained comparing pooled prevalence of the pulmonary veins variations in patients with and without AF. AF - atrial fibrillation. Non-AF - patients without atrial fibrillation.

Table 4 | Characteristics of each study included in diameter part of this meta-analysis.

Table 5 | Statistical results in diameter part of this meta-analysis. RSPV - right superior pulmonary vein. RIPV - right inferior pulmonary vein. LSPV - left superior pulmonary vein. LIPV - left inferior pulmonary vein. RMPV - right middle pulmonary vein. LCPV - left common pulmonary vein. CT - computed tomography. MRI - magnetic resonance imaging. MRA - magnetic resonance angiography. LCL - lower confidence interval. HCL - higher confidence interval.

Table 6 | Characteristics of each study included in ostial area part of this meta-analysis.

Table 7 | Statistical results in ostial area part of this meta-analysis. RSPV - right superior pulmonary vein. RIPV - right inferior pulmonary vein. LSPV - left superior pulmonary vein. LIPV - left inferior pulmonary 
vein. RMPV - right middle pulmonary vein. LCPV - left common pulmonary vein. CT - computed tomography. MRI - magnetic resonance imaging. MRA - magnetic resonance angiography. LCL - lower confidence interval. HCL - higher confidence interval.

\section{Hosted file}

PRISMA Diagram Flow (Figure 1).docx available at https://authorea.com/users/460312/articles/ 556351-variations-in-human-pulmonary-vein-ostia-morphology-a-systematic-review-with-

meta-analysis

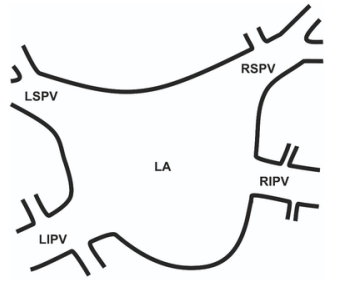

(A)

$70.25 \%$

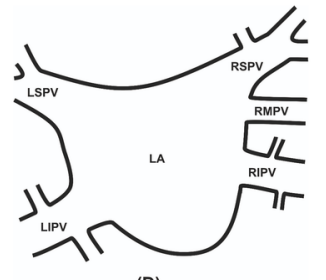

(D)

$8.38 \%$

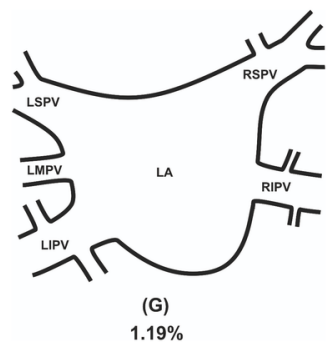

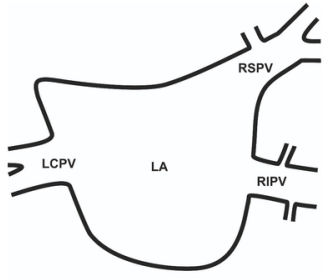

(B)

$17.37 \%$

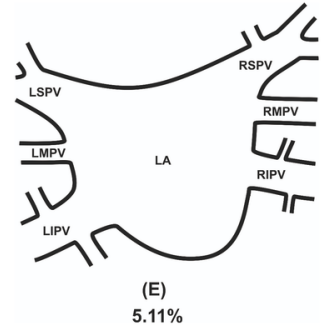

$5.11 \%$

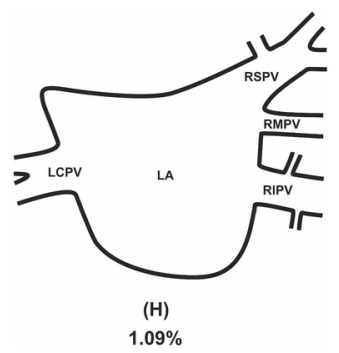

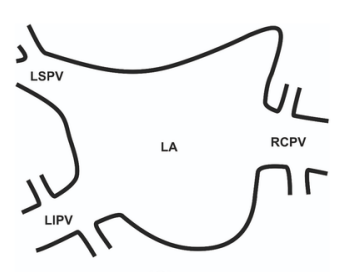

(C)

$8.78 \%$

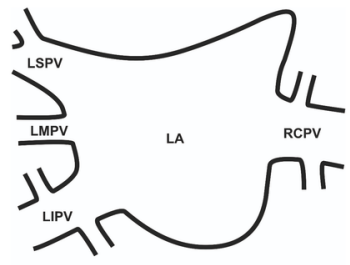

(F)

$1.24 \%$

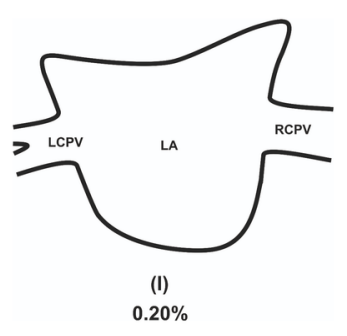

\section{Hosted file}

Table Characteristics Variations (Table 1).docx available at https://authorea.com/users/ 460312/articles/556351-variations-in-human-pulmonary-vein-ostia-morphology-a-systematicreview-with-meta-analysis

\section{Hosted file}

Table Variations (Table 2).docx available at https://authorea.com/users/460312/articles/ 556351-variations-in-human-pulmonary-vein-ostia-morphology-a-systematic-review-withmeta-analysis

\section{Hosted file}

AF vs Non-AF (Table 3).docx available at https://authorea.com/users/460312/articles/556351variations-in-human-pulmonary-vein-ostia-morphology-a-systematic-review-with-meta- 
analysis

\section{Hosted file}

Table Characteristics Diameter (Table 4).docx available at https://authorea.com/users/460312/ articles/556351-variations-in-human-pulmonary-vein-ostia-morphology-a-systematic-reviewwith-meta-analysis

\section{Hosted file}

Table \selectlanguage\{polish\}Ś \selectlanguage\{english\}rednica (Table 5).docx available https://authorea.com/users/460312/articles/556351-variations-in-human-pulmonary-veinostia-morphology-a-systematic-review-with-meta-analysis

\section{Hosted file}

Table Characteristics Ostial Area (Table 6).docx available at https://authorea.com/users/ 460312/articles/556351-variations-in-human-pulmonary-vein-ostia-morphology-a-systematicreview-with-meta-analysis

\section{Hosted file}

Table Ostial Area (Table 7).docx available at https://authorea.com/users/460312/articles/ 556351-variations-in-human-pulmonary-vein-ostia-morphology-a-systematic-review-withmeta-analysis 